\title{
Validación Preliminar de la Escala Infantil de Síntomas del Trastorno de Estrés Postraumático (Child PTSD Symptom Scale, CPSS) en Niños/as y Adolescentes Víctimas de Violencia Sexual
}

\section{Preliminary Validation of the Child PTSD Symptom Scale (CPSS) in Children and Adolescent Victims of Sexual Violence}

\author{
Patricia Bustos y Paulina Rincón \\ Universidad de Concepción
}

\author{
Jaime Aedo \\ Universidad Santo Tomás
}

\begin{abstract}
Se presenta la validación preliminar en Chile de la Escala Infantil de Síntomas del Trastorno de Estrés Postraumático, desarrollada por Foa, Johnson, Feeny y Treadwell (2001) para evaluar el trastorno en niños/as y adolescentes expuestos a situaciones traumáticas, con arreglo a criterios DSM-IV. La muestra fue de 75 niños y adolescentes chilenos de la región del Bío Bío que sufrieron abuso sexual o violación. Los resultados indican una alta consistencia interna, medida con alfa de Cronbach, de 0,916. Asimismo, la consistencia interna de cada subescala es alta. La validez convergente con el criterio de juicio experto es adecuada, con puntuaciones significativas en la escala y todas las subescalas.
\end{abstract}

Palabras clave: validación, trastorno de estrés postraumático, violencia sexual infantil.

\begin{abstract}
The preliminary validation in Chile of the Infantile Scale of Symptoms of Post Traumatic Stress Disorder (CPSS) is presented. This instrument was developed by Johnson, Feeny, and Treadwell (2001) to evaluate PTSD in children and adolescents exposed to traumatic events, in accordance with DSM-IV criteria. The sample consisted of 75 Chilean children and adolescents of the Bío Bío region of Chile who suffered sexual abuse or rape. The results indicate high internal consistency, measured with Cronbach's alpha, of 0.916. The convergent validity with the criterion of expert judgment is also adequate, with significant punctuations in the scale and all the subscales.
\end{abstract}

Keywords: validation, post traumatic stress disorder, childhood sexual violence.

\section{Introducción}

A través de los años se ha otorgado una atención especial a situaciones de violencia sexual en niños/as y adolescentes. La apertura de programas terapéuticos, nuevas formas de intervención, legislación existente y una mirada actual, destacan la toma de conciencia respecto a la gravedad, impacto y repercusiones profundas para quienes la sufren. Tal interés y concientización en temas de abuso sexual infantil se debe a múltiples factores, entre los que destacan la existencia real de este tipo de violencia en la pobla- ción infanto-adolescente chilena y la importancia creciente en relación a la magnitud, impacto y tiempos de intervención para abordarla. De esta forma, el Servicio Nacional de Menores (SENAME, 2001, citado en SENAME, 2004) tenía en Mayo de 2001 en su sistema de protección, de un total de 57.957 niños/as, a 26.409 (45,6\%) como víctimas de abuso sexual. El Centro de Atención a Víctimas de Atentados Sexuales de la Policía de Investigaciones señala que en Chile se producen 20.000 delitos por abuso sexual al año, lo cual significa que cada 26 minutos ocurre una agresión de este tipo.

Patricia Bustos, Departamento de Psicología, Universidad de Concepción, Concepción, Chile.

Paulina Rincón, Departamento de Psicología, Universidad de Concepción, Concepción, Chile.

Jaime Aedo, Departamento de Psicología, Universidad Santo Tomás, Los Ángeles, Chile.

La correspondencia relativa a este artículo debe ser dirigida a Patricia Bustos Torres, Departamento de Psicología,

Universidad de Concepción, Barrio Universitario s/n, Concepción, Chile. E-mail: patriciab@laradio.cl

El artículo se basa en la tesis de la primera autora para optar al grado de Magíster en Psicología, Mención Salud, del

Departamento de Psicología de la Universidad de Concepción. 
Se destaca que por cada delito denunciado en Chile, existen siete casos que no llegan a conocimiento de los organismos correspondientes. Además, mientras más próxima es la relación con el agresor sexual, menor es la probabilidad de denunciar (Carabineros \& Policía de Investigaciones, 2005, citado en SENAME, 2007).

En el año 2002 la Organización Mundial de la Salud (Organización Panamericana de la Salud, 2003) definió la violencia sexual como "todo acto sexual, la tentativa de consumar un acto sexual, los comentarios o insinuaciones sexuales no deseados, o las acciones para comercializar o utilizar de cualquier otro modo la sexualidad de una persona mediante coacción por otra persona, independiente de la relación de esta con la víctima, en cualquier ámbito, incluidos el hogar y el lugar de trabajo" (p. 161). Implica una relación de sometimiento entre agresor y víctima, en la cual esta última rechaza el acto sexual de forma explícita o implícita, por estar incapacitada o por falta de discernimiento (Barudy, 1998; Cantón \& Cortés, 1997; Echeburúa, de Corral, Amor, Sarasua \& Zubizarreta, 1997; San Martín, Viera, Gutiérrez \& González, 2002; Sosa \& Capafons, 1996). Los actos de violencia sexual involucran no solo a la víctima sino también al entorno familiar, social, ético y jurídico (Barudy, 1998; San Martín et al., 2002).

La postura imperante entre los investigadores es que, en la mayoría de los casos estudiados, las agresiones sexuales infantiles constituyen una experiencia traumática con repercusiones negativas en el estado psicológico de quienes las padecen (Echeburúa \& Guerricaechevarría, 2000). La gravedad e impacto del abuso sexual infantil, junto a las múltiples manifestaciones sintomáticas y patológicas producidas, dan cuenta que prácticamente no hay área de la sintomatología que no haya sido asociada a una historia de abuso sexual o violación (Browne \& Finkelhor, 1986; Cuffe, Addy, Garrison, Waller, Jackson, McKeown \& Chilappagari, 1998;Kendall-Tacket,Williams \& Finkelhor, 1993; Sanmartín, 1999; SENAME, 2004; Sosa \& Capafons, 1996). Investigaciones muestran que al menos un
$80 \%$ de las víctimas de agresiones sexuales infanto-adolescentes sufren consecuencias psicológicas negativas y presentan efectos que pueden repercutir en la vida adulta de no recibir un tratamiento adecuado y oportuno (Barudy, 1998; Cantón \& Cortés, 1996; DiLillo, 2001; Echeburúa \& de Corral, 2006).

Según Echeburúa y Guerricaechevarría (2000), en esta etapa vital son más frecuentes los sentimientos de culpa y vergüenza tras la situación de abuso sexual. Los síntomas más frecuentemente encontrados son los referidos a la "conducta sexualizada", considerada como el síntoma más característico del abuso sexual infantil. Esta se encuentra en el 35 a $40 \%$ de los casos de agresiones; seguida por el trastorno de estrés postraumático (TEP) en el 21 a $48 \%$ de los casos estudiados (American Psychiatric Association [APA], 1994; Barudy, 1998; Cantón \& Cortés, 2000; DiLillo, 2001; Echeburúa \& Guerricaechevarría; Finkelhor \& Berliner, 1995; Kendall-Tacket et al., 1993; Linning \& Kearney, 2004; Montt \& Hermosilla, 2001; Plaza, 2005; Sanmartín, 1999; Sosa \& Capafons, 1996; Stevenson, 1999).

El TEP se define como un trastorno que se presenta cuando la persona ha sufrido agresión física o algún acontecimiento que representa una amenaza para su vida o su integridad física, o cuando ha sido testigo o ha sabido por personas cercanas de la existencia de este tipo de acontecimientos en otras personas. La reacción emocional experimentada debe implicar una respuesta de temor, desesperanza $u$ horror intensos. Los grupos sintomáticos son: reexperimentación, evitación e hiperactivación. Estos deben prolongarse por más de un mes desde el suceso traumático y provocar malestar clínico o deterioro en áreas importantes de la actividad de la persona (APA, 1994, 2000).

$\mathrm{El}$ abuso sexual ha sido reconocido como un trauma serio de la vida y un potenciador de síntomas de TEP (Breslau, Davis, Andreski \& Peterson, 1991; Cuffe et al., 1998; Famularo, Fenton, Kinscherff, Ayoub \& Barnum, 1994; Kiser, Heston, Millsap \& Pruitt, 1991; Zeledón, Arce, Mejía \& Naranjo, 2003), encontrándose asociaciones entre su vivencia y el desarrollo de TEP (Deblinger, 
Mcleer, Atkins, Ralphe \& Foa, 1989; Kiser et al.; Rodriguez, Ryan, Vande Kemp \& Foy, 1997; Widom, 1999).

Para llevar a cabo la detección oportuna del TEP es útil y necesaria la evaluación a través de pruebas objetivas, diseñadas especialmente para su detección temprana. Para ello se han confeccionado numerosos instrumentos en muchos países, incluyendo las pruebas de auto reporte y las entrevistas diagnósticas estructuradas (Horowitz, Wilner \& Álvarez, 1979). Revisiones recientes de la literatura indican la necesidad de mejorar los instrumentos de evaluación en esta área (Bethea, 1999; Echeburúa \& de Corral, 2006; Echeburúa et al., 1997; Foa et al., 2001).

Sobre la base de la revisión de múltiples instrumentos para evaluar sintomatología postraumática, Foa et al. (2001) crearon una escala para evaluar de manera oportuna y rápida TEP en niños y adolescentes. Se trata de la Escala Infantil de Síntomas del Trastorno de Estrés Postraumático (The Child PTSD Symptom Scale, CPSS). La CPSS incluye 17 ítems acerca de los síntomas postraumáticos que abarcan los tres grupos sintomáticos (reexperimentación, evitación y activación). Fue validada en niños sobrevivientes de un terremoto en California, Estados Unidos. El estudio mostró que la CPSS mide sintomatología postraumática y es fácil de aplicar.

Dada la creciente importancia de evaluar el TEP en niños y adolescentes víctimas de violencia sexual y la inexistencia de instrumentos validados en la población chilena, que además sean breves y de fácil aplicación, se decidió validar preliminarmente la CPSS en una muestra de niños/as y adolescentes de las comunas de Concepción, Talcahuano, Hualpén, Tomé, Penco, Coronel y San Pedro, es decir, establecer las propiedades psicométricas de la CPSS en la población descrita: confiabilidad, validez y capacidad de discriminación.

\section{Método}

\section{Participantes}

El muestreo fue intencionado por cuotas respecto de la población de niños, ni- ñas y adolescentes víctimas de agresiones sexuales ingresados en centros especializados de la red SENAME de las provincias de Concepción y Bío Bío, Chile.

La muestra estuvo constituida por 75 niños/as y adolescentes víctimas de violencia sexual, en peritaje o tratamiento en esos centros, tanto Centros de Diagnóstico Ambulatorio (DAM) como Programas Especializados en Maltrato (PEM), y ciertos hogares para niños/as en esta situación. Las cuotas se definieron en función del origen de los casos, es decir, dependiendo del centro en el que se atendía a la población. Los criterios de inclusión fueron: a) tener entre $8 \mathrm{y}$ 18 años y b) haber sido víctima de violencia sexual, con presencia de informe sexológico forense que indicara lesiones atribuibles a violencia sexual o peritaje de veracidad de testimonio que calificara el relato como creíble. Fue criterio de exclusión presentar algún grado de retardo mental o analfabetismo. La evaluación se realizó entre Enero y Junio de 2008.

La edad promedio de la muestra alcanzó los 12,15 años $(D E=2,8)$; el $25,3 \%$ eran hombres y el $74,7 \%$, mujeres.

En cuanto a la historia de abuso, el $64 \%$ de los participantes había vivido abuso sexual y el $36 \%$, violación. El agresor se encontraba con mayor frecuencia en el núcleo intrafamiliar (63\%), seguido del agresor sexual extrafamiliar conocido (25\%) y, por último, el agresor extrafamiliar desconocido (12\%). De los niños/as ingresados a alguno de los centros especializados, el $75 \%$ recibía tratamiento reparatorio al momento de la develación y derivación a los centros respectivos.

Un $63 \%$ de los participantes contaba con pruebas periciales relativas a la constatación de lesiones y veracidad de testimonio de manera conjunta, un $13 \%$ contaba únicamente con pruebas periciales relativas a la constatación de lesiones, un $17 \%$ contaba solo con pruebas de veracidad de testimonio y un $7 \%$ no contaba con ninguna prueba pericial. Esto último se debe a que la historia de los niños que ingresaron por abuso sexual correspondía a tocaciones, por lo cual los profesionales no tenían mayor información pericial, sino solo la correspondiente a cambios evidentes de conducta y al relato de los niños/as o adolescentes, otorgando credibilidad al relato (Tabla 1). 
Tabla 1

Características de la Violencia Sexual en la Muestra

\begin{tabular}{llrr}
\hline Variable de Historia de Abuso Sexual & \multicolumn{1}{c}{ Categorías } & $n$ & \multicolumn{1}{c}{$\%$} \\
\hline \multirow{2}{*}{ Tipo de agresión sexual } & Violación & 27 & 36,0 \\
& Abuso sexual & 48 & 64,0 \\
\multirow{2}{*}{ Tipo de agresor } & Intrafamiliar & 47 & 62,7 \\
& Extrafamiliar conocido & 19 & 25,3 \\
& Extrafamiliar desconocido & 9 & 12,0 \\
Recibe tratamiento tras agresión & Sí & 56 & 74,7 \\
& No & 19 & 25,3 \\
Agresor vive con el niño/a o adolescente & Sí & 3 & 4,0 \\
& No & 72 & 96,0 \\
& Constatación de lesiones y & 47 & 62,7 \\
Pruebas periciales & credibilidad de testimonio & 10 & 13,3 \\
& Constatación de lesiones & 13 & 17,3 \\
& Credibilidad de testimonio & 5 & 6,7 \\
& Sin pruebas periciales & 18 & 24,0 \\
Tiempo transcurrido entre agresión y & 6 meses o menos & 27 & 36,0 \\
aplicación CPSS & Entre 6 meses y un año & 19 & 25,3 \\
& Entre 1 y 2 años & 11 & 14,7 \\
\hline
\end{tabular}

\section{Instrumentos}

Escala Infantil de Sintomas del Trastorno de Estrés Postraumático (CPSS). La CPSS es un instrumento desarrollado para evaluar la presencia de síntomas del Trastorno de Estrés Postraumático en niños/as y adolescentes de 8 a 18 años de edad con una historia conocida de trauma. La Escala está basada en los criterios diagnósticos del DSM-IV y se compone de 17 ítems con respuesta tipo Likert referidos a la frecuencia de manifestación de síntomas de este trastorno. Este instrumento puede ser utilizado como autoinforme o como entrevista clínica estructurada (Foa et al., 2001).

La CPSS se compone de 3 subescalas: Reexperimentación (5 ítems), Evitación (7 ítems) y Aumento de la Activación (5 ítems).

Las propiedades psicométricas de la versión original de la CPSS indican niveles aceptables de confiabilidad por consistencia interna, presentando coeficientes alfa de
Cronbach de 0,89 para la escala total, 0,80 para Reexperimentación, 0,73 para Evitación y 0,70 para Activación.

La confiabilidad, en términos de estabilidad temporal (correlación test-retest), alcanzó niveles aceptables para la escala completa de síntomas $(r[75]=0,84)$ y para las subescalas de Reexperimentación ( $r$ [75] $=0,85)$ y Activación $(r[75]=0,76)$. Con respecto a la subescala de Evitación, la estabilidad temporal alcanzó niveles relativamente bajos $(r[75]=0,63)^{1}$.

En cuanto a la validez convergente, en el estudio original se correlacionaron los puntajes de la CPSS con los de la Escala para Estrés Post Traumático en Niños (CPTSD, construida por Frederik et al. en 1985 y basada en los criterios diagnósticos para TEP del DSM-III) (Foa et al., 2001). La correlación fue de $r(75)=0,80, p<0,001)$.

\footnotetext{
1 En el estudio no se reporta el valor de $p$.
} 
Ficha de antecedentes personales. Se elaboró una ficha de antecedentes personales para recabar información pertinente respecto a antecedentes sociodemográficos y de historia de violencia sexual de los participantes.

Escalas de intensidad de sintomas para validez de criterio. Dado que no se encontraron instrumentos de medición del TEP estandarizados para la población infantil chilena, se usó como criterio externo de validación la evaluación de este trastorno por parte de jueces expertos que calificaron a cada evaluado con un puntaje respecto de la severidad de los síntomas de TEP en una escala de cuatro niveles (nula, baja, moderada y alta), de acuerdo a los criterios del DSM-IV.

\section{Procedimiento}

Selección de la escala. Se revisaron diversos instrumentos para evaluar TEP en niños/as y adolescentes. No obstante, las propiedades psicométricas de la gran mayoría de ellos son discutibles, otros son excesivamente largos, no entregan medidas de la severidad del TEP y/o no incluyen ítems que evalúen el daño funcional relacionado con el trauma vivido. Se eligió la CPSS por sus adecuadas propiedades psicométricas, porque evalúa presencia o ausencia de TEP y severidad del mismo y porque es una escala de rápida y fácil aplicación.

Adaptación de la versión en español de la Escala CPSS. Para la adaptación del instrumento, en primer lugar, se realizaron las modificaciones idiomáticas a la versión en español de la Escala ya existente, para adaptarla al castellano usado en Chile. Esta versión se obtuvo por contacto vía correo electrónico con los autores. Se trata solo de una traducción que no cuenta con un estudio psicométrico. Fue revisada por tres jueces expertos, quienes compararon la versión en inglés y la nueva versión en castellano. A estos jueces se les solicitó revisar el lenguaje de la Escala y proponer sugerencias, de modo que resultara comprensible para la población infanto-juvenil chilena. Tras ello, se realizaron las modificaciones indicadas.

Aplicación piloto de la escala. Se llevó a cabo una aplicación piloto de la escala a
12 niños/as y adolescentes ingresados en un DAM para evaluación pericial de veracidad de testimonio como supuestas víctimas de agresiones sexuales y a 10 niños/as y adolescentes de una escuela básica, con el propósito que señalaran si tanto las instrucciones como los ítems eran comprensibles para ellos. A la luz de estos resultados se hicieron nuevos cambios en la Escala.

Selección de los participantes. Se realizaron reuniones con los profesionales a cargo en las distintas instituciones, entregándoseles por escrito los objetivos de la investigación y analizando los aspectos éticos de la misma. Fueron los directores, consultando a los equipos técnicos de cada centro, quienes dieron el consentimiento informado para aplicar la Escala a los participantes de la investigación, ya que la información obtenida sería de utilidad en su diagnóstico o tratamiento.

Para establecer la muestra de niños/as y adolescentes víctimas de abusos sexuales o violación, se acudió a las fichas clínicas de los centros correspondientes, garantizando el anonimato y la confidencialidad de la información recabada.

Capacitación de los psicólogos expertos para la validez de criterio. Se llevó a cabo una capacitación para los psicólogos que actuarían como jueces para la validez de criterio. Los requisitos para actuar como juez experto fueron ser psicólogo con al menos dos años de experiencia en el área forense y estar a cargo de la atención del menor, es decir, haber establecido un vínculo previo con el evaluado que favoreciera la entrega de información y que evitara la victimización secundaria al exponer a los niños/as y adolescentes a la evaluación de otros profesionales.

Aplicación de los instrumentos de evaluación. La aplicación se llevó a cabo de manera individual y tardó, en promedio, 15 minutos por participante. Fue condición al momento de aplicar el instrumento que los niños/as y adolescentes se encontraran a solas con los psicólogos expertos, con el fin de evitar que sus respuestas fueran inducidas por terceros. La CPSS fue aplicada directa- 
mente por los profesionales, quienes previamente debían haber contestado la escala de validez de criterio, para no ver contaminadas sus respuestas con las de los niños/as y adolescentes. La corrección de la CPSS la realizó la primera autora de este artículo.

\section{Resultados}

\section{Adaptación de la Escala}

La CPSS fue elaborada en inglés, pero se disponía de una traducción al castellano. Sin embargo, esta versión traducida presentaba diferencias con el castellano utilizado en Chile. Por lo anterior, se realizaron modificaciones cuidando los siguientes aspectos: a) resguardar con la nueva pregunta el mismo ámbito sintomatológico al que estaba referida la pregunta original, y b) mantener la forma simple de preguntar y facilidad de comprensión, considerando sobre todo la población objetivo. Todas las modificaciones de redacción fueron resultado de la evaluación de la escala por parte de lo jueces expertos y de la aplicación piloto.

Es de destacar la modificación en el número de categorías de respuesta de la Escala. Tras considerar que el instrumento se refiere a sintomatología de las últimas dos semanas, se modificó el formato de respuestas de la escala Likert y se agregó una categoría más, dado que la primera categoría era nunca o solo una vez. En el espacio de dos semanas en el cual el instrumento hace el registro de información, se considera que es clínicamente distinto presentar los síntomas solo una vez en lugar de nunca. Se estima que los autores originales eligieron este tipo de alternativa al verse obligados a simplificar el formato de respuesta, considerando que el instrumento estaba diseñado para ser autoaplicado. En el entendido que la presente adaptación está dirigida a trabajar con el instrumento en un formato heteroaplicado, se decidió permitir a los evaluadores poder realizar discriminaciones más finas respecto de la frecuencia sintomática. Desde el punto de vista psicométrico, considerando que el modelo bajo el cual está diseñado la CPSS es el de la Teoría Clásica de los Test, el incre- mento del número de opciones de respuesta permite maximizar la confiabilidad del instrumento, sin afectar la predicción de las variables-criterio (Kramp, 2006).

Los ítems 1 a 17, en su versión original y modificada, junto a las categorías de respuesta, se presentan en la Tabla 2.

\section{Análisis Preliminar de los Resultados}

Para examinar potenciales diferencias en la manifestación de síntomas de TEP de acuerdo a la edad de los evaluados, se dividió la muestra en dos grupos de edad a partir de los 12 años, del mismo modo como se realizó en la construcción original de la CPSS.

$\mathrm{Al}$ igual que en la CPSS original, no se encontraron diferencias significativas $(0,27<p$ $<0,80$ ) en función de la edad de los evaluados respecto de los puntajes totales de la CPSS y de sus respectivas subescalas (Tabla 3 ).

Con respecto al sexo de los evaluados, no se encontraron diferencias significativas $(0,59<p<0,83)$ respecto de los puntajes totales de la CPSS y de sus respectivas subescalas (Tabla 4), a diferencia de lo ocurrido con la versión original de la CPSS, en la que los evaluados de sexo masculino presentaron puntuaciones significativamente inferiores a las evaluadas de sexo femenino (Foa et al., 2001).

\section{Puntajes de la CPSS}

Los promedios y desviaciones estándar de los puntajes de la versión adaptada de la CPSS y de sus tres subescalas fueron calculados para la muestra total, para la submuestra calificada por los jueces expertos con TEP nulo o bajo y para la submuestra calificada por los jueces expertos con TEP moderado o alto.

En la Tabla 5 pueden observarse las medias y desviaciones estándar de la CPSS para la muestra total y para las submuestras. Las medias de los puntajes de la Escala CPPS y de las tres subescalas son significativamente superiores en el grupo calificado por los jueces expertos como con alto TEP, en comparación con el grupo calificado con bajo TEP $(-6,44<t[73]<-5,47, p=0,000)$. 
Tabla 2

\section{Escala CPSS Original y Modificada}

Original

1. Has tenido pensamientos o imágenes molestas sobre el trauma aunque tú no querías pensar en eso.

\section{Has tenido sueños malos o pesadillas.}

3. Has actuado o has sentido como si el trauma estuviera pasando de nuevo (has escuchado algo o visto algo que te recuerda del trauma y te has sentido como si el trauma estuviera pasando otra vez).

4. Te has sentido mal cuando piensas o escuchas algo sobre el trauma (por ejemplo: sintiéndote asustado(a), enojado(a), triste o culpable).

5. Has tenido sensaciones en tu cuerpo cuando piensas o escuchas algo acerca del trauma (por ejemplo: sudando de repente, el corazón palpitando rápido).

6. Has tratado de no pensar, hablar, o de tener sentimientos acerca del trauma.

7. Has tratado de evitar actividades, personas, o lugares que te hacen recordar el evento traumático (por ejemplo, no querer jugar afuera o ir a la escuela).

8. Has tenido dificultad en recordar una parte importante del trauma.

9. Has tenido mucho menos interés o no has hecho las cosas que acostumbrabas hacer.

10. Has tenido dificultad en sentirte cercano a las personas que están a tu alrededor.

11. Has tenido dificultad en tener sentimientos fuertes (por ejemplo, no poder llorar o sentirte muy feliz).

12. Has sentido como si tus planes del futuro o tus esperanzas no se harán realidad (por ejemplo, no te graduarás de la escuela, no tendrás un trabajo, o no te casarás o no tendrás hijos(as).

13. Has tenido dificultad en quedarte o mantenerte dormido(a).

14. Te has sentido irritable o has tenido momentos de enojo.

15. Has tenido dificultad en concentrarte (por ejemplo, se te olvida de lo que se trata algún programa de la televisión, olvidándote de lo que leíste, o no prestando atención en la clase).

16. Has estado demasiado cuidadoso(a) y atento(a), (por ejemplo, cuando alguien camina detrás de ti).

17. Te has asustado o te has sorprendido fácilmente.
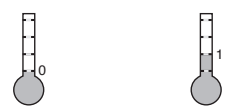

0

1
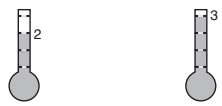

3

\begin{tabular}{|c|c|c|c|}
\hline $\begin{array}{c}\text { Nunca o solo } \\
\text { una vez }\end{array}$ & $\begin{array}{l}\text { De vez en } \\
\text { cuando }\end{array}$ & $\begin{array}{l}\text { La mitad del } \\
\text { tiempo }\end{array}$ & Casi siempre \\
\hline $\begin{array}{c}\text { Nunca o solo } \\
\text { una vez }\end{array}$ & $\begin{array}{l}\text { De vez en } \\
\text { cuando }\end{array}$ & $\begin{array}{l}\text { La mitad del } \\
\text { tiempo }\end{array}$ & Casi siempre \\
\hline $\begin{array}{c}\text { Nunca o solo } \\
\text { una vez }\end{array}$ & $\begin{array}{c}\text { De vez en } \\
\text { cuando }\end{array}$ & $\begin{array}{l}\text { La mitad del } \\
\text { tiempo }\end{array}$ & Casi siempre \\
\hline $\begin{array}{c}\text { Nunca o solo } \\
\text { una vez }\end{array}$ & $\begin{array}{c}\text { De vez en } \\
\text { cuando }\end{array}$ & $\begin{array}{c}\text { La mitad del } \\
\text { tiempo }\end{array}$ & Casi siempre \\
\hline $\begin{array}{c}\text { Nunca o solo } \\
\text { una vez }\end{array}$ & $\begin{array}{c}\text { De vez en } \\
\text { cuando }\end{array}$ & $\begin{array}{c}\text { La mitad del } \\
\text { tiempo }\end{array}$ & Casi siempre \\
\hline $\begin{array}{c}\text { Nunca o solo } \\
\text { una vez }\end{array}$ & $\begin{array}{c}\text { De vez en } \\
\text { cuando }\end{array}$ & $\begin{array}{l}\text { La mitad del } \\
\text { tiempo }\end{array}$ & Casi siempre \\
\hline $\begin{array}{c}\text { Nunca o solo } \\
\text { una vez }\end{array}$ & $\begin{array}{l}\text { De vez en } \\
\text { cuando }\end{array}$ & $\begin{array}{l}\text { La mitad del } \\
\text { tiempo }\end{array}$ & Casi siempre \\
\hline $\begin{array}{c}\text { Nunca o solo } \\
\text { una vez }\end{array}$ & $\begin{array}{c}\text { De vez en } \\
\text { cuando }\end{array}$ & $\begin{array}{c}\text { La mitad del } \\
\text { tiempo }\end{array}$ & Casi siempre \\
\hline $\begin{array}{c}\text { Nunca o solo } \\
\text { una vez }\end{array}$ & $\begin{array}{c}\text { De vez en } \\
\text { cuando }\end{array}$ & $\begin{array}{l}\text { La mitad del } \\
\text { tiempo }\end{array}$ & Casi siempre \\
\hline $\begin{array}{c}\text { Nunca o solo } \\
\text { una vez }\end{array}$ & $\begin{array}{l}\text { De vez en } \\
\text { cuando }\end{array}$ & $\begin{array}{l}\text { La mitad del } \\
\text { tiempo }\end{array}$ & Casi siempre \\
\hline $\begin{array}{c}\text { Nunca o solo } \\
\text { una vez }\end{array}$ & $\begin{array}{c}\text { De vez en } \\
\text { cuando }\end{array}$ & $\begin{array}{l}\text { La mitad del } \\
\text { tiempo }\end{array}$ & Casi siempre \\
\hline $\begin{array}{c}\text { Nunca o solo } \\
\text { una vez }\end{array}$ & $\begin{array}{c}\text { De vez en } \\
\text { cuando }\end{array}$ & $\begin{array}{l}\text { La mitad del } \\
\text { tiempo }\end{array}$ & Casi siempre \\
\hline $\begin{array}{c}\text { Nunca o solo } \\
\text { una vez }\end{array}$ & $\begin{array}{c}\text { De vez en } \\
\text { cuando }\end{array}$ & $\begin{array}{l}\text { La mitad del } \\
\text { tiempo }\end{array}$ & Casi siempre \\
\hline $\begin{array}{c}\text { Nunca o solo } \\
\text { una vez }\end{array}$ & $\begin{array}{c}\text { De vez en } \\
\text { cuando }\end{array}$ & $\begin{array}{l}\text { La mitad del } \\
\text { tiempo }\end{array}$ & Casi siempre \\
\hline $\begin{array}{c}\text { Nunca o solo } \\
\text { una vez }\end{array}$ & $\begin{array}{l}\text { De vez en } \\
\text { cuando }\end{array}$ & $\begin{array}{l}\text { La mitad del } \\
\text { tiempo }\end{array}$ & Casi siempre \\
\hline $\begin{array}{c}\text { Nunca o solo } \\
\text { una vez }\end{array}$ & $\begin{array}{c}\text { De vez en } \\
\text { cuando }\end{array}$ & $\begin{array}{l}\text { La mitad del } \\
\text { tiempo }\end{array}$ & Casi siempre \\
\hline $\begin{array}{c}\text { Nunca o solo } \\
\text { una vez }\end{array}$ & $\begin{array}{c}\text { De vez en } \\
\text { cuando }\end{array}$ & $\begin{array}{c}\text { La mitad del } \\
\text { tiempo }\end{array}$ & Casi siempre \\
\hline
\end{tabular}




\begin{tabular}{|c|c|c|c|c|c|}
\hline Modificada & & & & & \\
\hline \multirow[t]{2}{*}{ En las últimas 2 semanas....... } & . & 月. & $2^{2}$ & $7^{3}$ & \\
\hline & $\begin{array}{c}0 \\
\text { Nunca }\end{array}$ & $\begin{array}{l}1 \\
\text { Solo una } \\
\text { vez }\end{array}$ & $\begin{array}{c}2 \\
\text { Entre } 2 \text { y } \\
3 \text { veces }\end{array}$ & $\begin{array}{c}3 \\
\text { Entre } 4 \text { y } \\
8 \text { veces }\end{array}$ & $\begin{array}{l}4 \\
9 \text { veces y } \\
\text { más }\end{array}$ \\
\hline $\begin{array}{l}\text { 1. ¿Has tenido pensamientos o imágenes molestas acerca de lo } \\
\text { que te pasó y que vienen a tu mente aunque tú no hayas querido } \\
\text { recordarlas? }\end{array}$ & 0 & 1 & 2 & 3 & 4 \\
\hline 2. ¿Has tenido sueños malos o pesadillas? & 0 & 1 & 2 & 3 & 4 \\
\hline $\begin{array}{l}\text { 3. ¿Has tenido la sensación como si lo que te pasó estuviera } \\
\text { ocurriendo de nuevo, como si estuvieras ahí otra vez? (por } \\
\text { ejemplo, al escuchar o ver algo que te recuerde lo que a ti te pasó). }\end{array}$ & 0 & 1 & 2 & 3 & 4 \\
\hline $\begin{array}{l}\text { 4. ¿Te has sentido mal cuando piensas acerca de lo que te pasó o } \\
\text { cuando escuchas hablar acerca de lo que te pasó? (por ejemplo, } \\
\text { sintiéndote asustado, enojado, triste, culpable, con miedo, etc.). }\end{array}$ & 0 & 1 & 2 & 3 & 4 \\
\hline $\begin{array}{l}\text { 5. ¿Has tenido sensaciones en tu cuerpo cuando piensas o } \\
\text { escuchas acerca de lo que te pasó? (por ejemplo, transpirando } \\
\text { repentinamente o latiéndote muy rápido el corazón). }\end{array}$ & 0 & 1 & 2 & 3 & 4 \\
\hline $\begin{array}{l}\text { 6. ¿Haces esfuerzos para no pensar, no hablar o de no tener } \\
\text { sentimientos acerca de lo que te pasó? }\end{array}$ & 0 & 1 & 2 & 3 & 4 \\
\hline $\begin{array}{l}\text { 7. ¿Has tratado de evitar actividades, personas o lugares que te } \\
\text { recuerden lo que te pasó? }\end{array}$ & 0 & 1 & 2 & 3 & 4 \\
\hline 8. ¿Has olvidado una parte importante de lo que te pasó? & 0 & 1 & 2 & 3 & 4 \\
\hline $\begin{array}{l}\text { 9. ¿Has tenido muy pocas ganas de hacer cosas que antes } \\
\text { acostumbrabas hacer? }\end{array}$ & 0 & 1 & 2 & 3 & 4 \\
\hline $\begin{array}{l}\text { 10. ¿Has tenido dificultades para sentirte cercano(a) a tus seres } \\
\text { queridos? }\end{array}$ & 0 & 1 & 2 & 3 & 4 \\
\hline $\begin{array}{l}\text { 11. ¿Te cuesta mucho tener sentimientos fuertes? (por ejemplo, te } \\
\text { cuesta mucho llorar o sentirte feliz). }\end{array}$ & 0 & 1 & 2 & 3 & 4 \\
\hline $\begin{array}{l}\text { 12. ¿Has sentido que tus planes futuros o tus esperanzas no se } \\
\text { harán realidad? (por ejemplo, has sentido que no terminarás la } \\
\text { escuela, que no te casarás o que no tendrás hijos). }\end{array}$ & 0 & 1 & 2 & 3 & 4 \\
\hline $\begin{array}{l}\text { 13. ¿Has tenido problemas para quedarte dormido(a) o para } \\
\text { permanecer dormido(a) en la noche? }\end{array}$ & 0 & 1 & 2 & 3 & 4 \\
\hline 14. ¿Te enojas más fácilmente que antes de vivir lo que te pasó? & 0 & 1 & 2 & 3 & 4 \\
\hline $\begin{array}{l}\text { 15. ¿Has tenido dificultades para concentrarte? (por ejemplo, } \\
\text { perdiendo el hilo de una historia en la televisión, olvidando lo que } \\
\text { leías, no poniendo atención en clases). }\end{array}$ & 0 & 1 & 2 & 3 & 4 \\
\hline $\begin{array}{l}\text { 16. ¿Has estado demasiado vigilante? (por ejemplo, estás muy } \\
\text { atento(a) de que personas están a tu alrededor). }\end{array}$ & 0 & 1 & 2 & 3 & 4 \\
\hline $\begin{array}{l}\text { 17. ¿Te asustas más fácilmente que antes? (por ejemplo, cuando } \\
\text { alguien se acerca por detrás de ti). }\end{array}$ & 0 & 1 & 2 & 3 & 4 \\
\hline
\end{tabular}


Tabla 3

Medias y Desviaciones Estándar para la CPSS según Edad de los Evaluados

\begin{tabular}{|c|c|c|c|c|c|c|}
\hline \multirow{2}{*}{ CPSS } & \multicolumn{2}{|c|}{$\begin{array}{l}\text { Menores de } 12 \text { Años } \\
(n=41)\end{array}$} & \multicolumn{2}{|c|}{$\begin{array}{l}\text { Mayores de } 12 \text { Años } \\
\quad(n=34)\end{array}$} & \multirow{2}{*}{$t$} & \multirow{2}{*}{$p$} \\
\hline & Media & $\begin{array}{l}\text { Desviación } \\
\text { Estándar }\end{array}$ & Media & $\begin{array}{l}\text { Desviación } \\
\text { Estándar }\end{array}$ & & \\
\hline Total & 26,21 & 14,7 & 24,50 & 16,2 & 0,482 & 0,631 \\
\hline Reexperimentación & 8,20 & 4,8 & 6,91 & 5,3 & 1,094 & 0,278 \\
\hline Evitación & 10,10 & 7,0 & 9,35 & 6,5 & 0,475 & 0,636 \\
\hline Activación & 7,93 & 4,8 & 8,24 & 5,7 & $-0,254$ & 0,800 \\
\hline
\end{tabular}

Nota. Todas la pruebas de hipótesis de diferencia de medias fueron realizadas bajo el supuesto de homocedasticidad de las varianzas, confirmado mediante el test de Levene, con un nivel de significación 0,05.

Tabla 4

Medias y Desviaciones Estándar para la CPSS según Sexo de los Evaluados

\begin{tabular}{lcccccc}
\hline \multirow{2}{*}{ CPSS } & \multicolumn{2}{c}{$\begin{array}{c}\text { Femenino } \\
(n=56)\end{array}$} & \multicolumn{2}{c}{$\begin{array}{c}\text { Masculino } \\
(n=19)\end{array}$} & $t$ & $p$ \\
\cline { 2 - 5 } & Media & $\begin{array}{c}\text { Desviación } \\
\text { Estándar }\end{array}$ & Media & $\begin{array}{c}\text { Desviación } \\
\text { Estándar }\end{array}$ & & \\
\hline Total & 25,21 & 14,8 & 26,11 & 17,1 & $-0,218$ & 0,828 \\
Reexperimentación & 7,43 & 5,0 & 8,16 & 5,5 & $-0,540$ & 0,591 \\
Evitación & 9,64 & 6,6 & 10,11 & 7,3 & $-0,257$ & 0,798 \\
Activación & 8,14 & 4,9 & 7,84 & 6,0 & 0,216 & 0,829 \\
\hline
\end{tabular}

Nota. Todas la pruebas de hipótesis de diferencia de medias fueron realizadas bajo el supuesto de homocedasticidad de las varianzas, confirmado mediante el test de Levene, con un nivel de significación 0,05.

Tabla 5

Medias y Desviación Estándar de la CPSS para la Muestra Total y las Submuestras de Alto y Bajo TEP según Criterio de Jueces Expertos

\begin{tabular}{lccccccccc}
\hline \multirow{2}{*}{ CPSS } & \multicolumn{2}{c}{$\begin{array}{c}\text { Muestra total } \\
(n=75)\end{array}$} & \multicolumn{2}{c}{$\begin{array}{c}\text { Bajo TEP según } \\
\text { Criterio Jueces } \\
(n=35)\end{array}$} & $\begin{array}{c}\text { Alto TEP según } \\
\text { Criterio Jueces } \\
(n=40)\end{array}$ & $t$ & $p$ \\
\cline { 2 - 9 } & Media & $D E$ & Media & $D E$ & Media & $D E$ & & \\
\hline Total & 25,44 & 15,3 & 15,66 & 12,4 & 34,00 & 12,2 & $-6,444$ & 0,000 \\
Reexperimentación & 7,61 & 5,1 & 4,71 & 4,2 & 10,15 & 4,4 & $-5,469$ & 0,000 \\
Evitación & 9,76 & 6,7 & 5,88 & 5,6 & 13,15 & 5,8 & $-5,521$ & 0,000 \\
Activación & 8,07 & 5,2 & 5,06 & 4,2 & 10,70 & 4,6 & $-5,547$ & 0,000 \\
\hline
\end{tabular}

Nota. Todas la pruebas de hipótesis de diferencia de medias fueron realizadas bajo el supuesto de homocedasticidad de las varianzas, confirmado mediante el test de Levene, con un nivel de significación 0,05. 
Homogeneidad de los Ítems de la Versión Adaptada de la CPSS

Dado que la CPSS es un instrumento con un formato de respuestas de categorías ordenadas (tipo Likert o politómicas), y siguiendo lo señalado por López (2005), la estimación de los índices de homogeneidad se estableció a partir del uso del coeficiente de correlación producto-momento de Pearson entre el puntaje de cada ítem y la puntuación total del instrumento.

Como se muestra en la Tabla 6, todos los ítems del instrumento presentaron correlaciones directas y significativas $(p<$ $0,01)$ con el puntaje total del instrumento. Los coeficientes de correlación observados indican que cada ítem de la versión adaptada de la CPSS mide el mismo constructo evaluado por la escala total.

Tabla 6

Correlación Producto-Momento de Pearson entre el Puntaje de los Ítems y el Puntaje Total de la CPSS

\begin{tabular}{rcc}
\hline Ítem & $r$ Pearson Ítem-Test & $p$ \\
\hline 1 & 0,698 & 0,000 \\
2 & 0,610 & 0,000 \\
3 & 0,741 & 0,000 \\
4 & 0,711 & 0,000 \\
5 & 0,689 & 0,000 \\
6 & 0,685 & 0,000 \\
7 & 0,651 & 0,000 \\
8 & 0,371 & 0,001 \\
9 & 0,729 & 0,000 \\
10 & 0,693 & 0,000 \\
11 & 0,648 & 0,000 \\
12 & 0,598 & 0,000 \\
13 & 0,733 & 0,000 \\
14 & 0,679 & 0,000 \\
15 & 0,666 & 0,000 \\
16 & 0,597 & 0,000 \\
17 & 0,576 & 0,000 \\
\hline$n=75$ & &
\end{tabular}

Validez de los Ítems de la Versión Adaptada de la CPSS

Para establecer la validez de los ítems se calculó la correlación de rangos de Spearman entre el puntaje de cada ítem y las puntuaciones en TEP asignadas a los participantes por los profesionales expertos que oficiaron de criterio externo de validación.

Como se muestra en la Tabla 7, todos los ítems del instrumento presentaron correlaciones directas y significativas $(p \leq 0,022)$ con los puntajes del criterio externo de validación. Los datos expuestos dan cuenta de una adecuada validez de los ítems de la versión adaptada de la CPSS.

Tabla 7

Correlación de Rangos de Spearman entre el Puntaje de los Ítems y el Puntaje de TEP Asignado según Criterio Externo

\begin{tabular}{ccc}
\hline Ítem & $r$ Spearman Ítem-Criterio & $p$ \\
\hline 1 & 0,506 & 0,000 \\
2 & 0,460 & 0,000 \\
3 & 0,447 & 0,000 \\
4 & 0,537 & 0,000 \\
5 & 0,503 & 0,000 \\
6 & 0,485 & 0,000 \\
7 & 0,523 & 0,000 \\
8 & 0,264 & 0,022 \\
9 & 0,518 & 0,000 \\
10 & 0,354 & 0,002 \\
11 & 0,399 & 0,001 \\
12 & 0,399 & 0,000 \\
13 & 0,579 & 0,000 \\
14 & 0,441 & 0,000 \\
15 & 0,383 & 0,001 \\
16 & 0,410 & 0,000 \\
17 & 0,473 & 0,000 \\
\hline$n=75$ & & \\
& & \\
Confiabilidad de la Versión Adaptada de la \\
CPSS
\end{tabular}

Replicando lo realizado por los autores del instrumento, se estimó la confiabilidad del mismo, respecto de la consistencia interna, por medio del coeficiente alfa de Cronbach para las tres subescalas por separado y para la escala total.

Los coeficientes alfa de Cronbach oscilaron entre 0,78 y 0,92 (Tabla 8), siendo muy similares a los señalados respecto del instrumento original (Foa et al., 2001). Los datos obtenidos permiten establecer que el 
Tabla 8

Coeficientes Alfa de Cronbach para la Versión Original y la Versión Adaptada, según Escala Total y Subescala

\begin{tabular}{lccc}
\hline CPSS & $\begin{array}{c}\text { Número de } \\
\text { Ítems }\end{array}$ & $\begin{array}{c}\text { Alfa de Cronbach } \\
\text { Versión Adaptada } \\
\text { Chile }(N=75)\end{array}$ & $\begin{array}{c}\text { Alfa de Cronbach } \\
\text { Versión Original } \\
\text { EEUU }(N=75) \text { (Foa et } \\
\text { al., 2001) }\end{array}$ \\
\hline Escala Total & 17 & 0,916 & 0,89 \\
Reexperimentación & 5 & 0,842 & 0,80 \\
Evitación & 7 & 0,811 & 0,73 \\
Activación & 5 & 0,781 & 0,70 \\
\hline
\end{tabular}

instrumento adaptado presenta un aceptable nivel de confiabilidad por consistencia interna.

\section{Validez de la Versión Adaptada de la CPSS}

En este proceso de adaptación se utilizaron dos procedimientos para la estimación de la validez del instrumento, a saber, validez concurrente y análisis discriminante.

Validez concurrente. Siguiendo lo señalado por Anastasi y Urbina (1998), la validez concurrente "es adecuada para pruebas que se emplean para diagnosticar el estado actual, más que para predecir los resultados futuros" (p. 119).

En este caso se utilizó como criterio externo de validación concurrente el juicio clínico de un profesional experto. La correlación de rangos de Spearman entre el puntaje CPSS y el criterio externo de validación fue 0,71 ( $n=175, p=0,000)$, lo que permite aseverar que la versión adaptada de la CPSS resulta una medida adecuada de estimación del TEP en menores de edad.

Análisis discriminante. Este análisis se llevó a cabo considerando como variables independientes los puntajes en las tres subescalas (reexperimentación, evitación y activación) de la CPSS y como variable de agrupación, la calificación de severidad de síntomas asignada por los jueces expertos, dicotomizada de acuerdo al siguiente criterio: a) Bajo TEP: puntuaciones de sintomatología TEP nula o baja, b) Alto TEP: puntuaciones de sintomatología TEP mo- derada o alta.

A partir de los datos descritos, se obtuvo una correlación canónica de 0,74 , a la que corresponde un $\lambda=0,454\left(\chi^{2}[3, N=75]\right.$ $=56,403, p<0,0001)$. De estos valores se deduce que la versión adaptada de la CPSS discrimina significativamente entre quienes presentan un TEP alto o bajo, según la clasificación de los jueces expertos.

Se estableció, además, que a partir de las puntuaciones de las tres subescalas de la CPSS es posible clasificar correctamente al $90,7 \%$ de los casos clasificados previamente por los jueces expertos.

Con respecto al puntaje de corte, este se estableció visualmente en 24 puntos, luego de inspeccionar la distribución de los puntajes de la Escala total para los evaluados calificados con alto o bajo TEP de acuerdo al criterio de los jueces expertos. A partir de este punto de corte, los puntajes se distribuyen de acuerdo a lo señalado en la Tabla 9. De estos datos se infiere una sensibilidad de la prueba del $80 \%$ y una especificidad del $90 \%$.

\section{Conclusiones}

Este estudio piloto tuvo por objetivo verificar preliminarmente las características psicométricas de la adaptación chilena de la CPSS para población infantil y adolescente de la región del Bío Bío.

La adaptación que se está llevando a cabo con la CPSS la convertiría en el único instrumento en Chile diseñado específicamente para la evaluación del TEP de acuerdo a criterios DSM-IV-TR.

El desarrollo de un instrumento de este tipo le proporcionaría al clínico puntajes re- 
Tabla 9

Clasificación de la Severidad de Sintomas TEP según Jueces Expertos y Clasificación de la Versión Adaptada de la CPSS (Punto de Corte: 24 Puntos)

\begin{tabular}{lccc}
\hline & $\begin{array}{c}\text { Alto TEP } \\
\text { Según Jueces } \\
\text { Expertos }\end{array}$ & $\begin{array}{c}\text { Bajo TEP } \\
\text { según Jueces } \\
\text { Expertos }\end{array}$ & Total \\
\cline { 2 - 4 } Puntaje CPSS $\geq 24$ puntos (Alto TEP) & 36 & 3 & 39 \\
Puntaje CPSS $<24$ puntos (Bajo TEP) & 9 & 27 & 36 \\
Total & 45 & 30 & 75 \\
\hline
\end{tabular}

feridos a la severidad del trauma para cada uno de los tres grupos sintomáticos: reexperimentación, evitación y activación. Otro uso relevante se vincula con la evaluación de daño emocional en el marco de pericias forenses respecto de niños/as y adolescentes víctimas de delitos violentos, contexto en el que un instrumento de este tipo puede ser de gran utilidad.

La estimación preliminar de las propiedades psicométricas de la versión chilena de la escala CPSS permite establecer que el proceso de adaptación no ha alterado las propiedades psicométricas del instrumento original respecto de su confiabilidad y validez.

Sin perjuicio de lo anterior, se estiman necesarias algunas consideraciones. En primer lugar, este estudio investigó las reacciones psicológicas de niños/as o adolescentes respecto a dos tipos de agresiones sexuales: abuso sexual y violación. Sería de interés que el uso de la CPSS fuera extendido a la población de niños/as y adolescentes que hayan estado expuestos a diversos acontecimientos traumáticos, por ejemplo, niños/as víctimas de otros delitos violentos, víctimas de accidentes de tráfico o catástrofes naturales. En segundo lugar, la muestra utilizada en el estudio fue pequeña $\mathrm{y}$ heterogénea con respecto al sexo y comunas de procedencia, por lo que solo se pudo establecer un puntaje de corte a partir de un análisis discriminante, dato que es provisorio hasta probar el instrumento en una muestra de mayor tamaño.

En suma, los resultados de este estudio se evalúan como satisfactorios. El desarrollo de esta versión adaptada de la CPSS debe continuar en la línea de la verificación de sus propiedades psicométricas en una muestra de mayor cobertura nacional, para concluir con la generación de baremos para la población infanto-juvenil de Chile.

\section{Referencias}

American Psychiatric Association (1994). Diagnostic and statistical manual of mental disorders $\left(4^{\text {th }}\right.$ ed.). Washington, DC: Autor.

American Psychiatric Association (2000). Diagnostic and statistical manual of mental disorders: DSM$I V-T R$ ( $4^{\text {th }}$ ed. text revision). Washington, DC: Autor.

Anastasi, A. \& Urbina, S. (1998). Test psicológicos. Ciudad de México: Prentice Hall.

Barudy, J. (1998). El dolor invisible de la infancia: una lectura ecosistémica del maltrato infantil. Barcelona: Paidós.

Bethea, L. (1999). Primary prevention of child abuse. American Family Physician, 59, 1577-1585.

Browne, A. \& Finkelhor, D. (1986). Impact of child sexual abuse: A review of the research. Psychological Bulletin, 99, 66-77.

Breslau, N., Davis, G. C., Andreski, P. \& Peterson, E. (1991). Traumatic events and post-traumatic stress disorder in an urban population of young adults. Archives of General Psychiatry, 48, 216222.

Cantón, J. \& Cortés, M. R. (1996). Malos tratos y abuso sexual infantil. Madrid: Siglo XXI.

Cantón, J. \& Cortés, M. R. (1997). Malos tratos y abuso sexual infantil. Causas, consecuencias $e$ intervención. Madrid: Siglo XXI.

Cantón, J. \& Cortés, M. R. (2000). Guía para la evaluación del abuso sexual infantil. Madrid: Pirámide.

Cuffe, S. P., Addy, C. L., Garrison, C. Z., Waller, J. L., Jackson, K. L., McKeown, R. E. \& Chilappagari, S. (1998). Prevalence of PTSD in a community sample of older adolescents. Journal of the American Academy of Child \& Adolescent Psychiatry, 37, 147-154.

Deblinger, E., Mcleer, S. V., Atkins, M. S., Ralphe, D. \& Foa, E. (1989). Post-traumatic stress in sexually 
abused, psysically abused, and non abused children. Child Abuse \& Neglect, 13, 403-408.

DiLillo, D. (2001). Interpersonal functioning among women reporting a history of childhood sexual abuse: Empirical findings and methodological issues. Clinical Psychology Review, 21, 553-576.

Echeburúa, E., de Corral, P., Amor, P. J., Sarasua, B. \& Zubizarreta, I. (1997). Repercusiones psicopatológicas de la violencia doméstica en la mujer: un estudio descriptivo. Revista de Psicopatología y Psicología Clínica, 2, 7-19.

Echeburúa, E. \& Guerricaechevarría, C. (2000). Abuso sexual en la infancia: víctimas y agresores. Barcelona: Ariel.

Echeburúa, E. \& de Corral, P.(2006). Secuelas emocionales en víctimas de abuso sexual en la infancia. Cuadernos de Medicina Forense, 43-44, 75-82.

Famularo, R., Fenton, T., Kinscherff, R., Ayoub, C. \& Barnum, R. (1994). Maternal and child posttraumatic stress disorder in cases of child maltreatment. Child Abuse \& Neglect, 18, 27-36.

Finkelhor, D. \& Berliner, L. (1995). Research on the treatment of sexually abused children: A review and recommendations. Journal of the American Academy of Child \& Adolescent Psychiatry, 34, 1408-1423.

Foa, E., Johnson, K., Feeny, N. \& Treadwell, K. (2001). The Child PTSD Symptom Scale: A preliminary examination of its psychometric properties. Journal of Clinical Child Psychology, 30, 376384.

Horowitz, M., Wilner, N. \& Álvarez, W. (1979). Impacts of Event Scale: A measure of subjective stress. Psychosomatic Medicine, 41, 209-218.

Kendall-Tackett, K. A., Williams, L. M. \& Finkelhor, D. (1993). Impact of sexual abuse on children: A review and synthesis of recent empirical studies. Psychological Bulletin, 113, 164-180.

Kiser L. J., Heston, J., Millsap, P. A. \& Pruitt, D. B. (1991). Physical and sexual abuse in childhood: Relationship with post-traumatic stress disorder. Journal of the American Academy of Child \& Adolescent Psychiatry, 30, 776-783.

Kramp, U. (2006). Efecto del número de opciones de respuesta sobre las propiedades psicométricas de los cuestionarios de personalidad. Tesis no publicada para optar al grado de Doctor en Psicología Clínica i de la Salut, Universitat de Barcelona, España.

Linning, M. \& Kearney, C. (2004). Post-traumatic stress disorder in maltreated youth: A study of diagnostic comorbidity and child factors. Journal of Interpersonal Violence, 19, 1087-1101.

López, J. A. (2005). Ítems dicotómicos vs. politómicos: un estudio metodológico. Anales de Psicología, 21, 339-344.

Montt, M. E. \& Hermosilla, W. (2001). Trastorno de estrés post-traumático en niños. Revista Chilena de Neuro-Psiquiatría, 39, 110-120.

Organización Panamericana de la Salud (2003). La violencia sexual. En Organización Panamericana de la Salud, Informe mundial sobre la violencia y la salud (pp. 161-197). Extraído el 30 Abril, 2007, de http://www.paho.org/Spanish/AM/PUB/ capitulo_6.pdf

Plaza, I. (2005). El trastorno por estrés postraumático. Psicocentro. Extraído el 30 Abril, 2007, de http:/www.psicocentro.com/cgi-bin/articulo_ s.asp?texto=art32001

Rodriguez, N., Ryan, S. W., Vande Kemp, H. \& Foy, D. W. (1997). Posttraumatic stress disorder in adult female survivors of childhood sexual abuse: A comparison study. Journal of Consulting and Clinical Psychology, 65, 53-59.

Sanmartín, J. (1999). Violencia contra niños. Barcelona: Ariel.

San Martín, N., Viera, B., Gutiérrez, R. \& González, L. (2002). Violencia sexual en Chile y nueva ley de delitos sexuales. Fronteras en Obstetricia y Ginecología, 2(1), 61.

Servicio Nacional de Menores (2004). Estudio peritajes psicológicos en abuso sexual infantil. Santiago, Chile: Autor. Extraído el 17 Septiembre, 2007, de http://www.sename.cl

Servicio Nacional de Menores (2007). Estudio de prevalencia de violencia sexual y maltrato infantiladolescente. Santiago, Chile: Autor. Extraído el 17 Septiembre, 2007, de http://www.sename.cl

Sosa, S. \& Capafons, J. (1996). Abuso sexual en niños y adolescentes. En J. Buendía (Ed.), Psicopatología en niños y adolescentes. Desarrollos actuales (pp. 77-99). Madrid: Pirámide.

Stevenson, J. (1999). The treatment of the long-term sequelae of child abuse. The Journal of Child Psychology and Psychiatry, 40, 89-111.

Widom, C. S. (1999). Posttraumatic stress disorder in abused and neglected children grown up. The American Journal of Psychiatry, 156, 1223-1229.

Zeledón, C., Arce, J. G., Mejía, F. \& Naranjo, A. (2003). Trastorno de estrés postraumático en adolescentes agredidos. Medicina Legal de Costa Rica, 20(2), 15-25. 


\section{ANEXO}

ESCALA SEVERIDAD DE SÍNTOMAS PARA VALIDEZ DE CRITERIO

FECHA :

$\mathrm{ID}=$

CENTRO :

I.- Nombre del Niño/a, Adolescente:

II.- ¿Considera, de acuerdo a su experticia, que el niño/a o adolescente presenta Trastorno de Estrés Postraumático (TEP)?

Sí $\square \quad$ No $\square$

III.- La severidad de síntomas de TEP que el niño o adolescente presenta debido a la violencia sexual sufrida (abuso sexual o violación) es:
(3) Alta
(2) Moderada
(1) Baja
(0) Nula

IV.- NOMBRE PROFESIONAL REGISTRA:

Fecha de recepción: Julio de 2008.

Fecha de aceptación: Julio de 2009. 